\title{
Neuroendocrine immunity in patients with Alzheimer's disease: toward translational epigenetics
}

\author{
Francesco Chiappelli ${ }^{1 *}$, Paolo Prolo ${ }^{1}$, Kristine D. Cajulis ${ }^{1}$, Alberto Angeli ${ }^{2}$, Andrea Dovio ${ }^{2}$, Paola Perotti ${ }^{2}$, Marisa \\ Pautasso $^{3}$, Maria Luisa Sartori ${ }^{2}$, Laura Saba ${ }^{2}$, Stefano Mussino ${ }^{2}$, Thomas Fraccalini ${ }^{4}$, Fausto Fanto ${ }^{4}$, Ercolano Manfrini ${ }^{5}$, \\ Cristina Mocellini ${ }^{6}$, Maria Gabriella Rosso ${ }^{6}$, Enzo Grasso ${ }^{6}$ \\ ${ }^{1}$ Division of Oral Biology and Medicine, UCLA School of Dentistry, Los Angeles, CA 90095 1668, West-Los Angeles, Veterans Administration \\ Medical Center, Los Angeles, CA $90025 ;{ }^{2}$ Internal Medicine, University of Turin, A.S.O. S. Luigi Gonzaga, Orbassano, Italy; ${ }^{3}$ Flow Cytometry \\ Lab, University of Turin, A.S.O.S. Luigi Gonzaga, Orbassano, Italy; ${ }^{4}$ Geriatrics, University of Turin, A.S.O.S. Luigi Gonzaga, Orbassano, Italy; \\ ${ }^{5}$ Department of Neurology, University of Ancona, Italy; ${ }^{6}$ Neurology, A.S.O.S. Croce e Carle, Cuneo, Italy; Francesco Chiappelli Email: \\ chiappelli@dent.ucla.edu; Phone: 310794 6625; Fax: 310794 7901; * Corresponding author
}

\begin{abstract}
:
The emerging domain of epigenetics in molecular medicine finds application for a variety of patient populations. Here, we present fundamental neuroendocrine immune evidence obtained in patients with senile dementia of the Alzheimer's type (sDAT), and discuss the implications of these data from the viewpoint of translational epigenetics of Alzheimer's disease. We followed 18 subjects with mild sDAT treated with acetylcholinesterase inhibitors, and 10 control subjects matched for age in a repeated measure design every six months for 18 months. We monitored psychosocial profile (Mini-Mental State Examination, Functional Assessment Staging, Independence in Activities of Daily Living, Depression, Profile of Moods States) in parallel to immunophenotypic parameters of $\mathrm{T}$ cell subpopulations by flow cytometry. Based on change in the mini-mental state score at entry and at 18 months, patients with sDAT were assigned to a "fast progression" (delta greater than 2 points) or to a "slow progression" group (delta less than or equal to 2 points). The change in circulating activated T cells $(\mathrm{CD} 3+\mathrm{Dr}+)$ with time in patients with sDAT was significantly inversely correlated with the change in time in natural killer (NK) cytotoxic activity to cortisol modulation in these patients, which was greater in patients with fast progression, compared to slow progression sDAT. These data indicate underlying neuroendocrine immune processes during progression of sDAT. Our observations suggest that psychoimmune measures such as those we have monitored in this study provide relevant information about the evolving physiological modulation in patients with sDAT during progression of Alzheimer's disease, and point to new or improved translational epigenetic treatment interventions.
\end{abstract}

received April 14, 2007; accepted April 25, 2007; published online May 20, 2007

Key Words: Alzheimer's disease; neuroendocrine immunity; flow cytometry; epigenetics

\section{Background:}

Epigenetics refers to the domain of study of the molecular factors in the organism that modulate its adaptation to environment and intrinsic demands. [1] Epigenetic processes work in concert to enhance and to optimize physiological homeostasis and allostasis, the adaptation of the organisms following stress or pathologies. Molecular cartography, including genomics, proteomics, and interactomics, seeks to recognize and to identify the multi-faceted and intricate array of interacting genes and gene products that characterize the function and specialization of each individual cell in the context of cell-cell interaction, tissue, and organ function. Molecular epigenetics refers to the study of non-coding vs. coding DNA, and pertains to every domain of physiology, including the regulation of cellular immunity by specific $\mathrm{T}$ cell subpopulations (e.g., regulatory $\mathrm{T}$ cells, Tregs, CD4+CD25+FoxP3+ [2]), and the interaction between the immune and the neuroendocrine systems. [3]

Alzheimer's disease, a progressive neuro-degenerative disease caused in part by the deposit of the $\beta$ amyloid protein, is the fastest growing type of senile dementia. It is estimated that within the present decade, over five million people will be diagnosed with senile dementia of the Alzheimer's type (sDAT) in the US alone. Current treatment interventions include acetylcholinesterase inhibitors (e.g., Donezepil, [Aricept $\left.\left.{ }^{\circledR}\right]\right)$. [4-6]

Clinical observations indicate that patients with sDAT manifest marked immune disorders, which tend to worsen as the dementia progresses. Research data confirm this body of evidence, and indicate significantly impaired cell-mediated immune regulation, which appears to be on the one hand associated with altered $T$ cell-mediated immunity [3, 7], and on the other to be correlated with the severity of sDAT. $[8,9]$ Therefore, we tested the hypothesis that changes in the profile of circulating $\mathrm{T}$ cells is associated with neuroendocrine modulation of cellular immune function in these patients, and can have clinically relevant applicability as a prognostic factor of sDAT. The implications of our work pertains to the development of new and improved modes of treatment intervention for patients with sDAT that recognize and identify the multi-faceted and intricate array of both coding and non-coding DNA domains and gene products during the progression of Alzheimer's disease.

Description: A longitudinal prospective cohort study was designed to address the clinical applicability of physiologic modulation of cellular immune phenotypic and functional markers as prognostic factors of sDAT progression. In a repeated measure design, we followed 18 subjects (age range 
from 61 to 84 years old) with mild sDAT under treatment with acetylcholinesterase inhibitors (Donezepil $10 \mathrm{mg}$ per day), and 10 healthy control subjects (59 to 84 years of age). Patients were selected on the basis of short history of disease and no medication at baseline. Psychoimmune measures were obtained at entry and at six months intervals for 18 months.

At each visit, patients underwent a battery of psychometric tests [Mini-Mental State Examination (MMSE); Mini-Mental State Examination-age and education corrected (MMSE-C); Functional Assessment Staging (FAST); index of Independence in Activities of Daily Living (ADL); Center for Epidemiology Studies-Depression (CES-D); Profile of Moods States (POMS)] to evaluate their cognitive performance and their psychological status. Based on change in the MMSE score at entry and at 18 months follow-up, patients with sDAT were assigned to a "fast progression" $(\Delta$ $>2$ points; $\mathrm{n}=8$, two men, six women) or to a "slow progression" group $(\Delta \leq 2$ points; $(n=10$; two men, eight women).

We also examined in parallel immunophenotypic parameters of $\mathrm{T}$ cells by dual fluorescence flow cytometry. In brief, at each visit we obtained peripheral blood from an antecubital vein with ethylene diamine tetra-acetic acid (EDTA) as anticoagulant. Samples were collected between 0800 and 1000 hours, and the participants were instructed to take no food, caffeine, or nicotine for at least 1 hour prior to sample collection to minimize circadian and neuroendocrine-induced variability in immune measurements. Blood count (wbc) and differential were obtained, and the samples were centrifuged $(1500 \mathrm{rpm}$, room temp, $10 \mathrm{~min})$. Serum aliquots were cryopreserved at $-70^{\circ} \mathrm{C}$, and a fraction of the buffy coats used for whole blood flow cytometry. Flow cytometric data were analyzed by transforming the percent data produced by the flow analysis to absolute numbers using WBC and differential. [3] Flow cytometric data are presented in Table 1 (see supplementary material).

The remainder of the buffy coats was used for separation of peripheral blood mononuclear cells (PBMC) by standard buoyant density centrifugation. PBMC were tested for natural killer (NK) cytotoxicity of the human myeloblastoma cell line K562 target in the presence or the absence of the either negative (cortisol [Sigma, St. Louis, MO], micro M) or positive NK modulators (human recombinant IL-2 [Eurocetus, Emeryville, CA], $100 \mathrm{IU}$ per ml). NK cytotoxic activity was measured by the non-radiometric direct colorimetric 4-h assay, by assessing the concentration of lactate dehydrogenase (LDH) released by the lysed cells, and expressed as lytic units (LU) per 10 million cells. [10-14] NK modulation data are presented in Table 2 under supplementary material (note: the data in were published as original findings [14], and are presented here for reference purpose only.).

Data were expressed as means \pm standard deviation (SD), or standard error of the mean (SEM), as indicated. Parametric tests were used only when the data verified the three assumptions of normal distribution, independence of measurements and homogeneity of variance. Statistical significance of differences in the values of immune phenotype or function was determined by Student's t-test (or Wilcoxon's rank sign test). Repeated measure ANOVA (or Friedmann) was used to analyze the longitudinal data. Statistical analysis was performed at a level of significance $\alpha=0.10$ because of the variability accounted for by individual differences (Statistix [NIH Analytical Software, USA], Analyze-It, version 1.72 [Analyze-It Software, Ltd.]). [14]

Based on the change in MMSE from baseline, patients with sDAT were assigned to a "fast progression" group if the score $\Delta$ was greater than 2 points, or a "slow progression" group if the score $\Delta$ was less than or equal to 2 points. Patients with Fast Progression sDAT exhibited a significant drop in score of MMSE-C. Patients with sDAT manifested a significantly higher score on the CES-D scale, compared to control subjects (mean $\pm \mathrm{SD}$; control: $8.30 \pm 4.97$; slow progressing: $15.00 \pm 9.93, \mathrm{p}=0.08$; fast progressing: $14.40 \pm 6.26, \mathrm{p}=$ $0.03)$. These values did not change significantly in any of the groups at 6 months, 12 months or 18 months into the study (data not shown). POMS scores were higher, albeit not significantly, in patients with Fast Progression sDAT at entry (mean \pm SD: $28.33 \pm 23.29)$, compared to their cohorts with Slow progression sDAT symptoms $(16.14 \pm 31.56)$ and control subjects $(14.14 \pm 13.21)$. There was a notable lack of remarkable changes in CES-D and POMS among the groups across time.

It was also notable that the percent of CD3-CD16+CD56+ (phenotypic signature of NK cells) was indistinguishable among the three groups at entry (mean $\% \pm$ SD, control: $19.34 \pm 7.78$; Slow Progression sDAT: $15.2 \overline{5} \pm 7.32$; Fast Progression SDAT: $15.30 \pm 6.55)$, and remained comparable at 18 months (mean $\% \pm \mathrm{SD}$, control: $18.60 \pm 6.45$; Slow Progression sDAT: $13.6 \overline{6} \pm 7.71$; Fast Progression sDAT: $18.60 \pm 10.21)$. Patients with sDAT and normal control subjects also did not differ along $\mathrm{T}$ cell immunophenotypic indexes of CD4/CD8 ratio, CD4 $+\mathrm{T}$ cells, or $\mathrm{CD} 8+\mathrm{T}$ cells (Table 1 under supplementary material). Circulating activated $\mathrm{T}$ cells, recognized phenotypically as expressing the CD3 moiety $(\mathrm{CD} 3+)$ and the human leukocyte antigen $\mathrm{Dr}(\mathrm{Dr}+)$, did not change in the group of control subjects in time (mean $\pm \mathrm{SD}$; entry: $13.90 \pm 13.00,18$ month: $12.70 \pm 8.08$ ). However, the data showed a trend toward an increase in $\mathrm{CD} 3+\mathrm{Dr}+$ in patients with sDAT (mean $\pm \mathrm{SD}$; Slow Progression sDAT entry: $10.75 \pm 4.95 ; 18$ month: $14.13 \pm$ $5.87, \mathrm{p}=\mathrm{ns}$; Fast Progression sDAT entry: $13.10 \pm 7.89 ; 18$ month: $16.40 \pm 9.50, \mathrm{p}=\mathrm{ns})$.

The change in $\mathrm{CD} 3+\mathrm{Dr}+$ between entry and 18 month was not correlated with the change in MMSE score in patients with $\operatorname{sDAT}(r=0.012)$, but it was significantly inversely correlated with the change in time in the response of NK cytotoxic activity to cortisol modulation $(\mathrm{r}=-0.454, \mathrm{p}<0.05)$. This inverse relationship was stronger in the patients with Fast progression SDAT with 1.5 greater proportion of the variance accounted for by this correlative relationship, compared to patients with Slow progression sDAT. $\mathrm{CD} 3+\mathrm{Dr}+\mathrm{T}$ cells often express also the transferrin receptor, CD71, and the $\Delta$ chain of the IL-2 receptor, CD25. [15] These cells are endowed with the capacity toward end-stage 
maturation of T cells into mature memory cells capable of a TH1 or a TH2 pattern of cytokine production. CD25+ T cells are important regulatory cells (e.g., CD4+CD25+FoxP3+) of the cellular arm of immune surveillance. Our data suggest a trend toward an increase in Tregs in patients with sDAT. This observation is novel and critical, since the functional role of Tregs is to suppress activation of effector $T$ cells. If confirmed, this observation will shed light into the regulatory mechanisms of immune surveillance in patients with SDAT, and provide a better understanding of the clinical immunopathology of sDAT.

Our observations are also congruent with a putative intermediary role played by Tregs in SDAT. Cortisol in particular up-regulates IL-10 [16], a key regulatory cytokine of NK cells and of Tregs. At the molecular level, this regulatory process appears to be accounted for by the glucocorticoids-induced tumor necrosis factor receptor (TNFr) family-related gene (GITR) on Tregs. [17] The functional role of GITR in Tregs is likely to be associated with the signaling pathways involving the family of suppressors of cytokine signaling (SOCS). [18] The observation that IL-10 induces the expression of SOCS- 1 and SOCS-3, an outcome that is dependent upon activation of the signal transducer and activator of transcription (STAT) 3 by chain 1 of the IL-10 receptor (IL-10R1), and is associated with the inhibition of IFN $\gamma$ signaling [15] confirms the putative role of these pathways in the cortisol modulation of $\mathrm{NK}$, and other cellular immune functions. The next frontier in cellular immunology research in SDAT will include the characterization of the role of IL-10 and cortisol directly on the SOCS pathway in NK and other T cell sub-populations.

In other studies, we have discussed the concept of evidencebased translational research and practice as the 2-way transfer between fundamental investigative work and the clinical endeavor as it pertains directly to patients with Alzheimer's disease. We have noted that laboratory evidence, such as the reliable assessment of the $\beta$ amyloid protein in salivary samples [19], and psycho-cognitive and emotional assessment of fit and adaptation [20] must contribute to a well-rounded evidence-based clinical decision-making process.

Here, we propose the workable hypothesis that during progression of SDAT, characteristic shifts occur in the reading frame of the genome, therefore obfuscating normally coding DNA, and exposing otherwise non-coding DNA. That is to say, significant changes in the molecular epigenetic structure of the genome are likely to occur during progression of SDAT, which may lead to marked alterations in the molecular cartography inherent to a variety of cells, tissues and organs of the patient, including cells of the immune system. Consequently, Tregs and other T cell subpopulations, and NK cells, may be expected to find their capacity to respond to and to be modulated by neuroendocrine products progressively adulterated as the disease progresses. Further molecular research should be directed at confirming and expanding these observations, and at testing this fundamental hypothesis. Confirmatory studies of this type will uncover new avenues of molecular medicine interventions for patients with Alzheimer's disease grounded on translational evidencebased epigenetics.

Acknowledgment: This work was supported in part by Fondazione Cassa di Risparmio di Cuneo; Fondazione Cassa di Risparmio di Saluzzo; Wilshire Rotary Foundation of Los Angeles; NIDDKD P50 DK 64539; and the Alzheimer's Association. The Authors want to thank Daniela Baraban of the Flow cytometry Laboratory at Ospedale S.Luigi, Orbassano for her valuable contribution to this study.

\section{References:}

[01] I. Rigoutsos, et al., PNAS, 103:6605 (2006) [PMID: 16636294]

[02] S. Floess, et al., PloS Biol, 5:38 (2007) [PMID: 17298177]

[03] F. Chiappelli, et al., Alzheimer's Disease: New Frontiers for the 21st Century, Nova Science Publisher, 233 (2006)

[04] H. Fillit and J. Cummings, Manag care Interface, 13:51 (2000) [PMID: 10747691]

[05] J. L. Cummings, Clin Cornerstone, 3:27 (2001) [PMID: 11432120]

[06] F. Chiappelli, et al., Evid Based Complement Alternat Med., 3:411 (2006) [PMID: 17173104]

[07] E. Esumi, et al., Acta Neurol Scand., 84:65 (1991) [PMID: 1927262]

[08] M. Fiala, et al., Eur J Clin Invest., 32:360 (2002) [PMID: 12027877]

[09] M. Fiala, et al., J Alzheimer's Dis., 7:221 (2005) [PMID: 16006665]

[10] R. G. Masera, et al., Psychoneuroendocrinol., 27:447 (2002) [PMID: 11911998]

[11] A. Dovio, et al., Int $J$ Obes Relat Metab Disord., 28:894 (2004) [PMID: 15208649]

[12] C. Korzeniewski \& D. M. Callewaert, J. Immun Methods, 64:313 (1983) [PMID: 6199426]

[13] H. F. Pross, et al., J. Clin. Immunol., 1:51 (1981) [PMID: 7334070]

[14] P. Prolo, et al., Bioinformation., 1:363 (2007)

[15] F. Chiappelli, Encyclopeadia of Stress II, Elsevier, (2007)

[16] E. J. Peek, et al., Am J Respir Cell Mol Biol., 33:105 (2005) [PMID 15845862]

[17] K. Uraushihara, et al., J Immunol., 171:708 (2003) [PMID: 12847237]

[18] R. S. McHugh, et al., Immunity, 16:311 (2002) [PMID: 11869690]

[19] F. Chiappelli, et al., Bioinformation., 1:329 (2006)

[20] F. Chiappelli, et al., Ca Dent Assoc J., 34:439 (2006)[PMID: 16866013]

Edited by $P$. Kangueane

Citation: Chiappelli et al., Bioinformation 2(1): 1-4 (2007)

License statement: This is an open-access article, which permits unrestricted use, distribution, and reproduction in any medium, for non-commercial purposes, provided the original author and source are credited. 
www.bioinformation.net

\section{Current Trends}

\section{Supplementary material}

\begin{tabular}{|c|c|c|c|c|c|c|c|c|}
\hline \multicolumn{5}{|c|}{ Entry } & \multicolumn{4}{|c|}{18 month } \\
\hline $\begin{array}{l}\text { Sample } \\
\text { number }\end{array}$ & CD4+ & CD3+Dr+ & CD8+ & $\mathrm{CD} 4 / \mathrm{CD} 8$ & CD4+ & CD3+Dr+ & CD8+ & $\mathrm{CD} 4 / \mathrm{CD} 8$ \\
\hline \multicolumn{9}{|l|}{ Control } \\
\hline 1 & 45 & 2 & 18 & 2.5 & 46 & 4 & 16 & 2.87 \\
\hline 2 & 42 & 8 & 21 & 2.0 & 41 & 16 & 22 & 1.86 \\
\hline 3 & 41 & 13 & 26 & 1.06 & 37 & 10 & 27 & 1.4 \\
\hline 4 & 43 & - & 32 & 1.3 & 38 & 23 & 32 & 1.2 \\
\hline 5 & 50 & 11 & 21 & 2.3 & 53 & 9 & 20 & 2.6 \\
\hline 6 & 50 & 8 & 30 & 1.6 & 43 & 15 & 20 & 2.15 \\
\hline 7 & 54 & 4 & 27 & 1.9 & 52 & 2 & 28 & 1.8 \\
\hline 8 & 44 & 14 & 22 & 2.0 & 36 & 17 & 28 & 1.29 \\
\hline 9 & - & - & - & - & 36 & 26 & 37 & 0.97 \\
\hline 10 & 45 & 9 & 21 & 2.1 & 47 & 5 & 20 & 2.03 \\
\hline Mean & 42.60 & 13.90 & 26.60 & 1.86 & 42.90 & 12.70 & 25.00 & 1.82 \\
\hline SD & 11.51 & 13.00 & 8.75 & 0.46 & 6.40 & 8.08 & 6.50 & 0.62 \\
\hline \multicolumn{9}{|l|}{ Slow $\mathrm{P}^{1}$} \\
\hline 11 & 38 & 9 & 39 & 0.98 & 35 & 9 & 28 & 1.25 \\
\hline 12 & 44 & 10 & 38 & 1.15 & 45 & 11 & 33 & 1.4 \\
\hline 13 & 56 & 4 & 16 & 3.5 & 57 & 25 & 18 & 3.1 \\
\hline 14 & 37 & 15 & 19 & 1.95 & 38 & 15 & 22 & 1.73 \\
\hline 15 & 64 & 4 & 15 & 4.2 & 57 & 13 & 13 & 4.38 \\
\hline 16 & 50 & 15 & 17 & 2.9 & 52 & 16 & 19 & 2.7 \\
\hline 17 & 43 & 17 & 23 & 1.86 & 58 & 18 & 22 & 2.6 \\
\hline 18 & 48 & 12 & 23 & 2.01 & 50 & 6 & 27 & 1.85 \\
\hline Mean & 47.50 & 10.75 & 23.75 & 2.32 & 49.00 & 14.13 & 22.75 & 2.38 \\
\hline SD & 9.13 & 4.95 & 9.57 & 1.13 & 8.88 & 5.87 & 6.36 & 1.04 \\
\hline \multicolumn{9}{|l|}{ Fast $\mathrm{P}^{2}$} \\
\hline 19 & 44 & 10 & 21 & 2.0 & 46 & 14 & 20 & 2.02 \\
\hline 20 & 29 & 9 & 26 & 1.1 & 27 & 20 & 21 & 1.29 \\
\hline 21 & 44 & 25 & 28 & 1.57 & 44 & 38 & 30 & 1.47 \\
\hline 22 & 52 & 25 & 28 & 1.9 & 45 & 10 & 29 & 1.55 \\
\hline 23 & 50 & 11 & 24 & 2.0 & 45 & 5 & 23 & 1.96 \\
\hline 24 & 40 & 18 & 23 & 1.07 & 42 & 14 & 22 & 1.90 \\
\hline 25 & 42 & 1 & 27 & 1.5 & 52 & 14 & 18 & 2.9 \\
\hline 26 & 56 & 16 & 16 & 3.5 & 42 & 13 & 17 & 2.47 \\
\hline 27 & 42 & 4 & 24 & 1.75 & 40 & 10 & 23 & 1.7 \\
\hline 28 & 62 & 14 & 9 & 6.9 & 54 & 26 & 8 & 6.8 \\
\hline Mean & 46.10 & 13.10 & 22.60 & 2.33 & 43.70 & 16.40 & 21.10 & 2.41 \\
\hline SD & 9.27 & 7.89 & 6.00 & 1.74 & 7.32 & 9.50 & 6.23 & 1.62 \\
\hline
\end{tabular}

Table 1: Immunophenotypic characterization of the subjects at entry and 18 months (given as \% of circulating lymphocytes, identified by the CD45+CD14- LeukoGate gate) 1: Slow progression SDAT; 2: Fast progression sDAT

\begin{tabular}{|c|c|c|c|c|c|c|}
\hline \multicolumn{3}{|c|}{ Baseline } & \multicolumn{2}{|c|}{ Cortisol $\left(10^{-6} \mathrm{M}\right)$} & \multicolumn{2}{|c|}{ hrIL-2 (650 IU/ml) } \\
\hline & $\begin{array}{c}\text { Mean } \\
\text { (lytic units) }\end{array}$ & +SEM & $\begin{array}{c}\text { Mean } \\
\text { (lytic units) }\end{array}$ & +SEM & $\begin{array}{c}\text { Mean } \\
\text { (lytic units) }\end{array}$ & \pm SEM \\
\hline Control & 32.51 & 6.5 & 23.92 & 3.83 & 75.83 & 19.41 \\
\hline Slow Progressing sDAT ${ }^{1}$ & 23.26 & 3.31 & 15.89 & 2.38 & 79.39 & 28.50 \\
\hline p vs. control & 0.24 & & 0.10 & & 0.92 & \\
\hline Fast Progressing sDAT $^{1}$ & 21.13 & 3.40 & 15.33 & 2.60 & 38.46 & 6.82 \\
\hline p vs. control & 0.14 & & 0.08 & & 0.09 & \\
\hline
\end{tabular}

Table 2: Modulation of NK Activity (at entry) (1: as determined by drop in MMSE score at 18 months) 